\title{
Poly(ADP-ribose) polymerase-3 overexpression is associated with poor prognosis in patients with breast cancer following chemotherapy
}

\author{
ZHIGUO SONG ${ }^{1}$, YONG WANG ${ }^{2}$, QINGHUAN XIAO ${ }^{3}$, ZHAOJIN YU $^{1}$, LIN ZHAO $^{1}$, HUIZHE WU $^{1}$, \\ MINGLI SUN ${ }^{1}$, ZHANGGUO CHAI ${ }^{4}$, PING HOU ${ }^{5}$, XIAOQIANG GENG ${ }^{1}$, WENSI LIU ${ }^{1}$ and MINJIE WEI ${ }^{1}$ \\ ${ }^{1}$ Department of Pharmacology, School of Pharmacy, China Medical University, Shenyang, Liaoning 110122; \\ ${ }^{2}$ Department of General Practice, The First Hospital of China Medical University, Shenyang, Liaoning 110001; \\ ${ }^{3}$ Deparment of Ion Channel Pharmacology, School of Pharmacy, China Medical University, Shenyang, Liaoning 110122; \\ ${ }^{4}$ Outpatient Department, Shenyang Artillery Academy, Shenyang, Liaoning 110867; ${ }^{5}$ Liaoning Blood Center, \\ Shenyang, Liaoning 110044, P.R. China
}

Received October 17, 2015; Accepted May 18, 2017

DOI: $10.3892 / \mathrm{ol} .2018 .9398$

\begin{abstract}
Double strand breaks induced by genotoxic agents, if inappropriately repaired, will cause cell death or induce cancer. Poly(ADP-ribose) polymerase-3 (PARP-3) serves a role in double strand break repair, and may be involved in tumorigenesis. To the best of our knowledge, the role of PARP-3 in breast cancer has not yet been examined. In the present study, the expression of PARP-3 was investigated in 493 breast cancer samples and 54 tumor-adjacent control samples using tissue-microarray-based immunohistochemistry. PARP-3 expression was higher in breast cancer samples compared with control samples. PARP-3 overexpression was significantly associated with histological grade II-III $(\mathrm{P}=0.012)$. In addition, PARP-3 overexpression was significantly associated with shorter disease-free survival (DFS; $\mathrm{P}=0.027$ ) time and exhibited a tendency toward shorter overall survival (OS; $\mathrm{P}=0.183$ ) time in patients with breast cancer compared with patients with lower PARP-3 expression, particularly in BRCA1-positive patients $(\mathrm{P}=0.004$ for disease-free survival and $\mathrm{P}=0.095$ for OS). Multivariate Cox regression analysis indicated that PARP-3 was an independent prognostic factor in patients with breast cancer. Furthermore, it was revealed that PARP-3 overexpression was associated with shorter survival time in patients with cyclophosphamide/doxorubicin or epirubicin/5-fluorouracil (CAF/CEF) chemotherapy compared with low PARP-3 expression, but not in patients with $\mathrm{CAF} / \mathrm{CEF}+$ docetaxel chemotherapy. The present
\end{abstract}

Correspondence to: Dr Minjie Wei, Department of Pharmacology, School of Pharmacy, China Medical University, 77 Puhe Road, Shenyang North New Area, Shenyang, Liaoning 110122, P.R. China E-mail: weiminjiecmu@163.com

Key words: breast cancer, poly(ADP-ribose) polymerase, breast cancer gene 1 , chemotherapy, survival study suggested that PARP-3 may be used as a biomarker for predicting the clinical outcome of patients receiving chemotherapy, and targeting PARP-3 may be a potential therapeutic strategy for the treatment of breast cancer.

\section{Introduction}

Breast cancer is a group of heterogeneous diseases with different clinical and histological forms (1). The molecular and clinical heterogeneity of breast cancer renders it necessary to identify biomarkers of clinical outcomes so that patients can be treated with the most appropriate chemotherapeutic protocols (2). Therefore, identification of biomarkers that will predict breast cancer to chemotherapeutic drugs is important for the future development of individualized treatment for patients with breast cancer.

Poly(ADP-ribose) polymerases (PARPs) constitute a family of enzymes that catalyze poly(ADP-ribosylation) of DNA-binding proteins and perform a key role in the regulation of transcription, genome stability, energy metabolism, tumorigenesis and cellular responses to DNA damage $(3,4)$. The PARP superfamily is composed of 17 members, of which PARP-1, PARP-2 and PARP-3 are activated by DNA strand breaks and serve an important role in the repair of single strand breaks and/or double strand breaks (DSBs) (5-7). PARP-1, the most studied member of the PARP superfamily, has been reported to be overexpressed in numerous malignant tumors, including breast cancer, and is associated with invasiveness and poor clinical outcomes (8-13). In addition, it is well-known that the a combination of PARP inhibitors and DNA damaging chemotherapy, can increase tumor responses and improve the survival of triple-negative patients with breast cancer compared with chemotherapy alone (14). Most importantly, PARP inhibitors are particularly efficient against tumors with defects in DNA repair mechanisms, including tumors with breast cancer gene (BRCA) mutations $(15,16)$.

Despite having similar functions in the regulation of cellular responses to DNA damage, PARP-3 exhibits structural and 
functional differences from PARP-1 (17). Although PARP-3 shares a conserved $\mathrm{C}$-terminal region with PARP-1, it has a shorter N-terminal region than PARP-1. PARP-3 also exhibits different $\mathrm{N}$-terminal functions, including DNA binding or DNA-dependent activation (18). In addition, it has been reported that knockdown of PARP3, but not PARP1, results in an increase in the production of DSB induced by ionizing radiation (19). However, unlike PARP-1, less is known about the role of PARP-3 in breast cancer. Bieche et al (20) reported that the mRNA expression of PARP-3 was under-expressed in $10.4 \%$ of patients with breast cancer and this PARP-3 under-expression was mutually exclusive with overexpression of PARP-1. To date, the protein expression of PARP-3 in patients with breast cancer has not yet been investigated. It remains to be determined whether the protein level of PARP-3 is consistent with its mRNA level in breast cancer.

In the present study, the expression of PARP-3 was investigated in 493 breast cancer samples and 54 tumor-adjacent control samples using immunohistochemistry. The present study aimed to analyze the association of PARP-3 expression with clinicopathological features, chemotherapeutic responses and prognosis of patients with breast cancer.

\section{Patients and methods}

Ethics statement. The present study was approved by the Medical Ethics Committee of China Medical University (Shenyang, China). Due to the retrospective nature of the study, the Medical Ethics Committee waived the requirement of written informed consents by the patients.

Patients. The present study included human breast tissues from 493 female patients with breast cancer, who underwent surgery at the First Affiliated Hospital of China Medical University between January 2005 and October 2010. A total of 54 samples adjacent to the tumors outside the cancer loci were collected as controls. The diagnosis of breast cancer was confirmed by pathological staining. Histological evaluation of 54 samples adjacent to tumors exhibited no histological tumor-associated features.

The average age of patients with breast cancer was $51.3 \pm 10.6$ years (range, $20-82$ years). The histological grade of the cancer was determined according to the World Health Organization grading system $(21,22)$. The stage of the cancer was evaluated according to the tumor-node-metastasis (TNM) staging system (22). Clinicopathological data, including patient age, menopausal status, tumor size, lymph node metastasis, p53 status and BRCA1 status were retrospectively retrieved from medical records.

All patients did not undergo radiation therapy and chemotherapy prior to surgery. Following surgery, 291 patients received cyclophosphamide/doxorubicin or epirubicin/5-fluorouracil (CAF/CEF) and 95 patients received $\mathrm{CAF} / \mathrm{CEF}$ and docetaxel (CAF/CEF and D). The remaining 107 patients received other chemotherapeutic regimens containing docetaxel or cisplatin alone or in combination.

Immunohistochemistry. Tissue sections $(4 \mu \mathrm{m})$ were fixed with $4 \%$ formalin at room temperature for $48 \mathrm{~h}$ and paraffin-embedded tissue blocks for immunohistochemical staining. Sections were
Table I. Clinicopathological characteristics of patients with breast cancer.

Parameters Total, $\mathrm{n} \quad$ Patients, $\mathrm{n}(\%)$

Age at diagnosis

$\leq 51$ years

$>51$ years

Menopausal status

Pre-menopause

Post-menopause

Tumor size

$\leq 2.0 \mathrm{~cm}$

$>2.0,<5.0 \mathrm{~cm}$

$\geq 5.0 \mathrm{~cm}$

Tumor type

Ductal carcinoma

Lobular carcinoma

Mucinous carcinoma

Others

Histological grade

G1

$\mathrm{G} 2$

G3

TNM stage

I-II

III-IV

Lymph node metastasis

No

Yes

p53

Negative

Positive

BRCA1

Negative

Positive

Chemotherapy regimen

$\mathrm{CAF} / \mathrm{CEF}$

$\mathrm{CAF} / \mathrm{CEF}+\mathrm{T}$
493

$279(56.6)$

214 (43.4)

493

$268(54.4)$

225 (45.6)

493

$325(65.9)$

146 (29.6)

$22(4.5)$

492

455 (92.5)

$11(2.2)$

9 (1.8)

$17(3.5)$

83 (16.9)

367 (74.6)

42 (8.5)

493

364 (73.8)

129 (26.2)

493

268 (54.4)

225 (45.6)

$166(41.8)$

231 (58.2)

396

$102(25.8)$

294 (74.2)

386

291 (75.4)

95 (24.6)
CAF/CEF, cyclophosphamide/doxorubicin or epirubicin/5-fluorouracil; $\mathrm{CAF} / \mathrm{CEF}+\mathrm{T}, \mathrm{CAF} / \mathrm{CEF}+$ docetaxel; TNM, tumor-node-metastasis; BRCA1, breast cancer susceptibility gene 1 .

deparaffinized with xylene, rehydrated in a graded alcohol series of 100 and $95 \%$ (Sinopharm Chemical reagent Co., Ltd., Shanghei, China) at a concentration of $100,95,85,75,65 \%$ and $\mathrm{H}_{2} \mathrm{O}$ and sections were put into $3 \%$ citric acid-sodium citrate buffer $(\mathrm{pH}=6.0)$ and heated in a microwave oven at $100^{\circ} \mathrm{C}$ for $10 \mathrm{~min}$ to retrieve the antigen. Endogenous peroxidase activity was blocked by incubating the sections in $3 \% \mathrm{H}_{2} \mathrm{O}_{2}$ at $37^{\circ} \mathrm{C}$ for $20 \mathrm{~min}$. Sections were subsequently blocked to avoid nonspecific binding with $10 \%$ normal goat serum (Boster Biological Technology, Pleasanton, CA, USA) at $37^{\circ} \mathrm{C}$ for $30 \mathrm{~min}$ and 

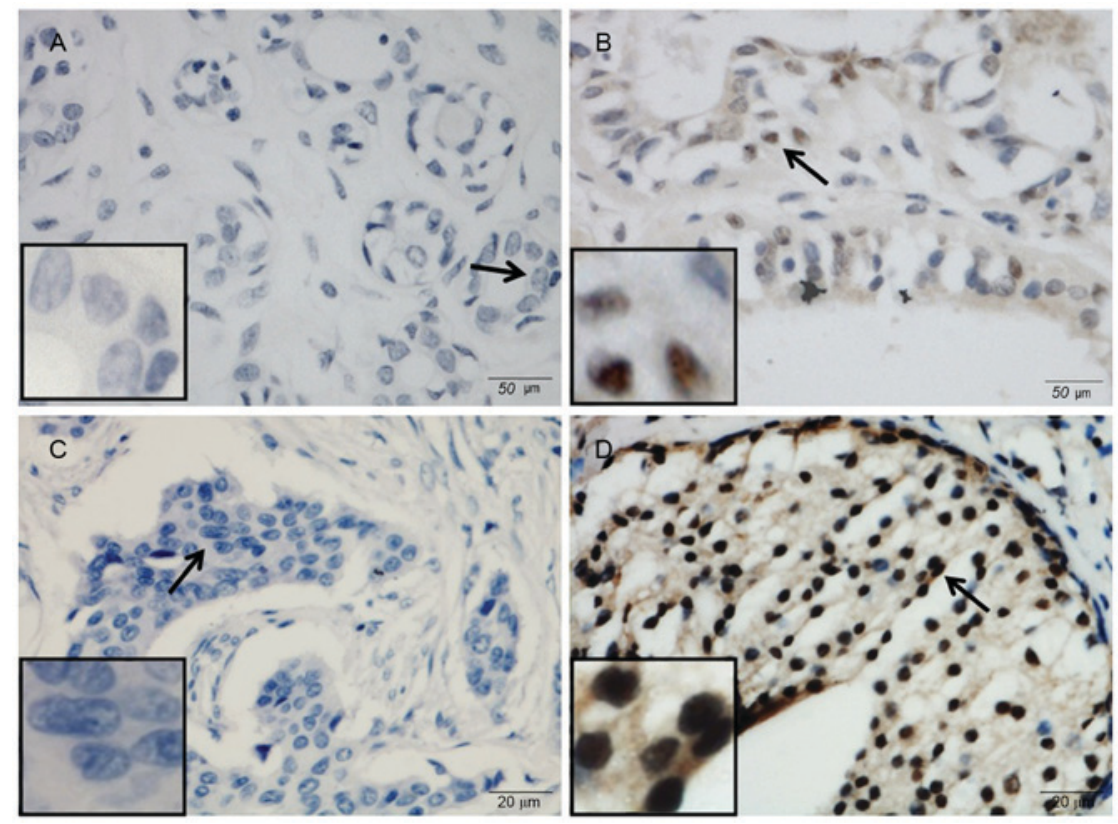

Figure 1. Representative micrographs showing (A and C) negative and (B and D) positive immunohistochemical staining of poly(ADP-ribose) polymerase-3 in (A and B) tumor-adjacent tissues and (C and D) breast cancer tissues. Arrows indicate the magnified regions in the insert. Magnification, $\mathrm{x} 400 . \mathrm{Scale}$ bar, $50 \mu \mathrm{m}$.

incubated at $4^{\circ} \mathrm{C}$ overnight with the polyclonal antibody against PARP-3 (dilution, 1:100; cat. no. 96601; rabbit anti-human polyclonal antibodies; Abcam, Cambridge, UK), followed by incubation with biotinylated secondary antibodies (secondary antibody $\mathrm{A}$ in the kit-0305; dilution, 1:200; cat. no. kit-0305; Maxim Biotechnologies, Fuzhou, China) for $30 \mathrm{~min}$ at $37^{\circ} \mathrm{C}$. Sections were then incubated with streptavidin horseradish peroxidase (secondary antibody B in the kit-0305; dilution, 1:200; cat. no. kit-0305; Maxim Biotechnologies) for an additional $20 \mathrm{~min}$ at $37^{\circ} \mathrm{C}$ and stained with 3,3-diaminobenzidine at room temperature for $1 \mathrm{~min}$ (dilution, 1:200; cat. no. LI-9032; OriGene Technologies, Inc, Beijing, China). Sections were counterstained with hematoxylin, dehydrated and mounted. For negative controls, the sections were not incubated with primary antibodies.

Evaluation of immunohistochemistry. The immunostained sections were examined under the light microscope (magnification, x200; select 3 fields/view) by two pathologists blinded to the experimental conditions. The intensity of immunoreactivity was scored as follows: 0, no staining; 1, weak staining; 2 , moderate staining and 3 , strong staining. A percentage scoring system was used to assess the number of stained cells and the scores were assigned by using $5 \%$ increments as previously reported $(23,24)$. The final scores were used to determine the cutoff value for discriminating tumors with the high expression of PARP-3 from tumors with the low expression, using receiver operating characteristic (ROC) curves. The sensitivity and specificity for the survival of patients with breast cancer was plotted to generate ROC curves.

Statistical analysis. Analyses were performed using SPSS 16.0 (SPSS, Inc., Chicago, IL, USA). Pearson's $\chi^{2}$ or Fisher's exact probability tests were used to evaluate the association between PARP-3 expression and clinicopathological characteristics of patients with breast cancer. Survival probabilities were estimated by the Kaplan-Meier method and assessed by a log-rank test. Univariate and multivariate Cox proportional hazards regression models were used for assessing the association between potential confounding variables and prognosis [overall survival (OS) or disease-free survival (DFS)]. OS was calculated as the time between the first day of diagnosis and disease-associated mortality or last known follow-up. The disease-free survival (DFS) was calculated as the time between the first day of diagnosis and the occurrence of local recurrence or distant metastasis. $\mathrm{P}<0.05$ was considered to indicate a statistically significant difference.

\section{Results}

Clinicopathological characteristics of patients with breast cancer. Table I summarizes clinicopathological characteristics of 493 patients with breast cancer. Of the 493 patients, age, menopausal status, tumor size, tumor type, histological grade, TNM stage and lymph node metastasis were recorded in 493, 493, 493, 492, 492, 493 and 493 patients, respectively. The majority of these patients had a tumor with invasive ductal carcinoma $(92.5 \%),<2 \mathrm{~cm}$ in size $(65.9 \%)$, histological grade II (74.6\%) or TNM stage I-II (73.8\%). Lymph node metastasis occurred in 225 (45.6\%) of 493 patients.

Follow-up information was available for 493 patients with breast cancer. During the follow-up period of 9-118 months, relapses occurred in 85 cases and cancer-associated mortalities were identified in 55 cases. The 5-year survival rate was $88.0 \%$. The mean OS and DFS times were 66.3 and 63.7 months, respectively.

PARP-3 overexpression in breast cancer. The expression of PARP-3 was studied in 493 breast cancer samples and 54 tumor-adjacent control samples using immunohistochemistry (Fig. 1). PARP-3 was mainly expressed in the nucleus. Nuclear expression of PARP-3 was observed in 234 (47.5\%) of 

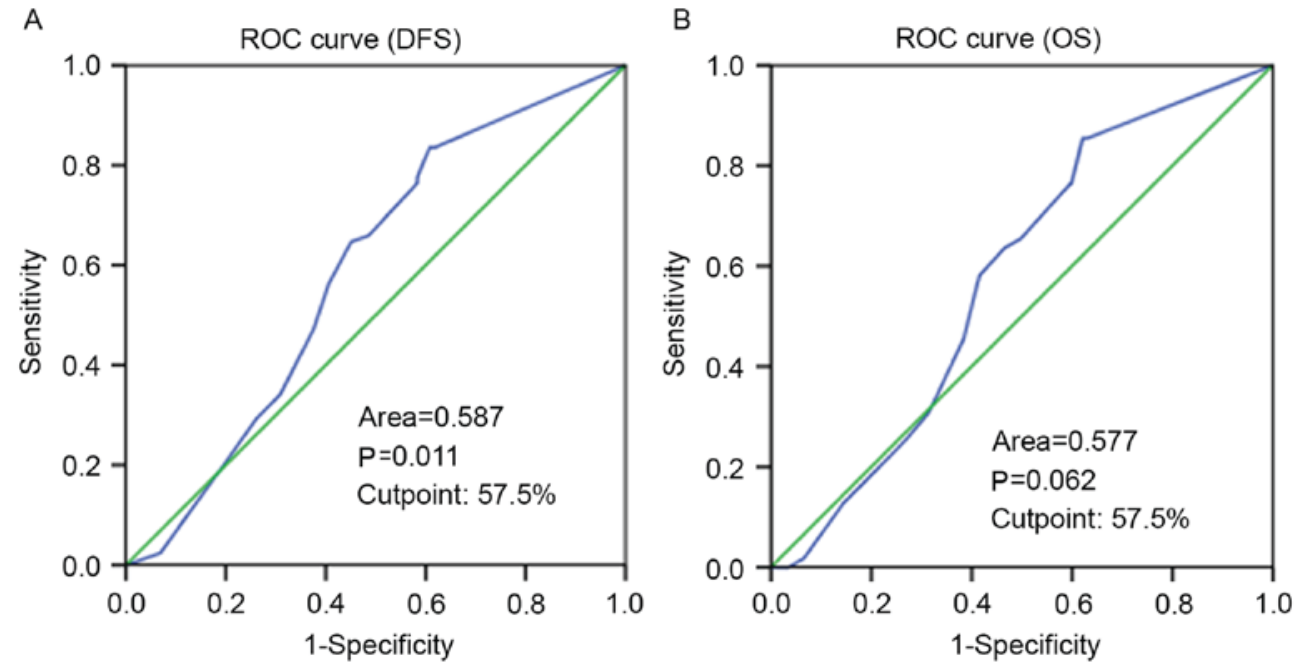

Figure 2. ROC curves were used to determine the cutoff score for poly(ADP-ribose) polymerase-3 overexpression in breast cancer, based on the DFS of patients with breast cancer. Values for sensitivity and specificity for (A) DFS and (B) OS were plotted. The areas under the curve and P-value are indicated in the figure. ROC, receiver operating characteristic; DFS, disease-free survival; OS, overall survival.

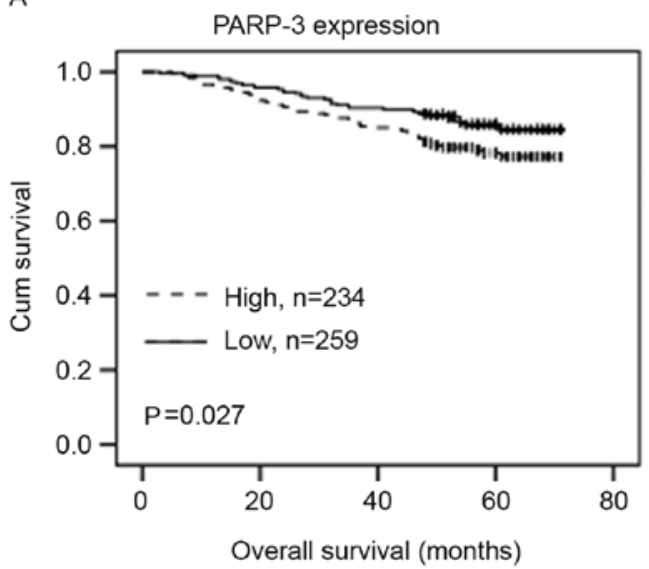

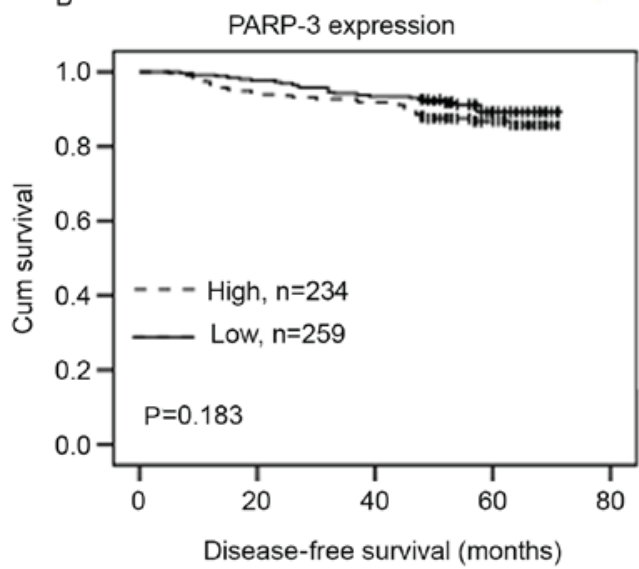

Figure 3. Kaplan-Meier survival analysis of PARP-3 expression in patients with breast cancer. The log-rank test was performed to test statistical significance. Survival curves show the association between PARP-3 expression and (A) DFS or (B) OS in 493 patients with breast cancer with PARP-3 expression. PARP-3, poly(ADP-ribose) polymerase-3; DFS, disease-free survival; OS, overall survival; Cum, cumulative.

493 breast cancer samples and $9(16.7 \%)$ of 54 control samples. PARP-3 immunoreactivity occurred significantly more frequently in breast cancer samples compared with control samples $(\mathrm{P}<0.001)$.

Selection of the cutoff value for PARP-3 expression. ROC curve analysis was performed to determine an optimal cutoff score for PARP-3 expression in breast cancer samples. Based on DFS time data, a cutoff score of $57.5 \%$ was selected for PARP-3 expression (Fig. 2). Tumors with immunohistological scores $\geq 57.5$ and $<57.5 \%$ were defined as tumors with high and low PARP-3 expression, respectively. A total of 234 (47.5\%) tumors exhibited high expression and 259 (52.5\%) tumors showed low expression.

Association of PARP-3 expression with clinicopathological characteristics of patients with breast cancer. The association between PARP-3 expression and clinicopathological characteristics of patients with breast cancer was investigated (Table II).
Age, menopausal status, tumor size, tumor type, TNM stage, lymph node metastasis, p53 status and BRCA1 status were not significantly associated with the expression of PARP-3. High PARP-3 expression level was associated with histological grade II-III $(\mathrm{P}=0.012)$ when compared with PARP-3 low expression level.

Association of PARP-3 expression with the survival of patients with breast cancer. The association of the PARP-3 expression with the OS or DFS in patients with breast cancer was evaluated using Kaplan-Meier analysis and log-rank test. PARP-3 overexpression was significantly associated with shorter DFS time ( $\mathrm{P}=0.027)$ (Fig. 3A). Although PARP-3 expression exhibited a tendency toward shorter OS, no statistically significant difference was observed $(\mathrm{P}=0.183$ ) (Fig. 3B).

The association of PARP-3 expression with the OS or DFS in breast cancer patients with different BRCA1 statuses was then investigated. In BRCA1-negative patients, PARP-3 expression was not significantly associated with the OS or 
Table II. Association between PARP-3 expression and clinicopathological features of patients with breast cancer.

\begin{tabular}{|c|c|c|c|}
\hline \multirow[b]{2}{*}{ Characteristics } & \multicolumn{2}{|c|}{$\begin{array}{c}\text { PARP-3 } \\
\text { expression, } \mathrm{n}(\%)\end{array}$} & \multirow[b]{2}{*}{ P-value ${ }^{a}$} \\
\hline & High & Low & \\
\hline \multicolumn{4}{|l|}{ Age at diagnosis } \\
\hline$\leq 51$ years & $138(49.5)$ & $141(50.5)$ & 0.310 \\
\hline$>51$ years & $96(44.9)$ & $118(55.1)$ & \\
\hline \multicolumn{4}{|l|}{ Menopausal status } \\
\hline Pre-menopause & $133(49.6)$ & $135(50.4)$ & 0.294 \\
\hline Post-menopause & $101(44.9)$ & $124(55.1)$ & \\
\hline \multicolumn{4}{|l|}{ Tumor size } \\
\hline$\leq 2.0 \mathrm{~cm}$ & $156(48.0)$ & $169(52.0)$ & 0.942 \\
\hline$>2.0,<5.0 \mathrm{~cm}$ & $68(46.6)$ & $78(53.4)$ & \\
\hline$\geq 5.0 \mathrm{~cm}$ & $10(45.5)$ & $12(54.5)$ & \\
\hline \multicolumn{4}{|l|}{ Lymph node metastasis } \\
\hline No & $122(45.5)$ & $146(54.5)$ & 0.346 \\
\hline Yes & $112(49.8)$ & $113(50.2)$ & \\
\hline \multicolumn{4}{|l|}{ TNM stage } \\
\hline I-II & $175(48.1)$ & $189(51.9)$ & 0.647 \\
\hline III-IV & $70(54.3)$ & $59(45.7)$ & \\
\hline \multicolumn{4}{|l|}{ Histological grade } \\
\hline G1 & $29(34.9)$ & $54(65.1)$ & 0.012 \\
\hline $\mathrm{G} 2$ & $178(48.5)$ & $189(51.5)$ & \\
\hline G3 & $26(61.9)$ & $16(38.1)$ & \\
\hline \multicolumn{4}{|l|}{ Histological type } \\
\hline Ductal carcinoma & $216(47.5)$ & $239(52.5)$ & 0.196 \\
\hline Lobular carcinoma & $7(63.6)$ & $4(36.4)$ & \\
\hline Mucinous carcinoma & $6(66.7)$ & $3(33.3)$ & \\
\hline Other & $5(29.4)$ & $12(70.6)$ & \\
\hline \multicolumn{4}{|l|}{ p53 status } \\
\hline Negative & $80(48.2)$ & $86(51.8)$ & 0.650 \\
\hline Positive & $106(45.9)$ & $125(54.1)$ & \\
\hline \multicolumn{4}{|l|}{ BRCA1 status } \\
\hline Negative & $52(51.0)$ & $50(49.0)$ & 0.289 \\
\hline Positive & $132(44.9)$ & $162(55.1)$ & \\
\hline
\end{tabular}

ap-value calculated using $\chi^{2}$ or Fisher's exact. PARP-3, poly(ADP-ribose). polymerase-3; TNM, tumor-node-metastasis; BRCA1, breast cancer susceptibility gene 1 .

DFS ( $\mathrm{P}>0.05$ ) (Fig. 4A and B). However, in BRCA1-positive patients, PARP-3 expression exhibited a tendency toward shorter OS, but this association was not statistically significant $(\mathrm{P}=0.095)$ (Fig. 4C). PARP-3 overexpression was significantly associated with shorter DFS time ( $\mathrm{P}=0.004)$ (Fig. 4D). Furthermore, there was a greater association between the combination of high PARP3 and BRCA1 expression and shorter OS $(\mathrm{P}=0.055)$ (Fig. 4E) and DFS time $(\mathrm{P}=0.002)$ (Fig. 4F) compared with non-combination of PARP3 and BRCA1.

Univariate Cox regression analysis was performed to estimate the impact of each clinicopathological variable on
OS and DFS in patients with breast cancer. The univariate analysis identified that menopausal status [hazard ratio $(\mathrm{HR})=1.527 ; \mathrm{P}=0.044]$, histological grade $(\mathrm{HR}=2.674$; $\mathrm{P}=0.012)$, TNM stage $(\mathrm{HR}=4.20 ; \mathrm{P}<0.001)$ and lymph node metastasis $(\mathrm{HR}=2.513 ; \mathrm{P}<0.001)$ were significantly associated with the OS and DFS of patients with breast cancer (Table III). Age $(\mathrm{P}=0.041)$ and tumor size $(\mathrm{P}=0.042)$ were also identified to be significantly associated with the OS of patients with breast cancer. In addition, PARP-3 overexpression was significantly associated with shorter DFS time of patients with breast cancer $(\mathrm{P}=0.029$; Table III). Furthermore, multivariate Cox regression analysis (Table IV) indicated that TNM stage $(\mathrm{RR}=5.665 ; \mathrm{P}<0.001)$, menopausal status $(\mathrm{RR}=2.535 ; \mathrm{P}=0.045)$ and $\mathrm{PARP}-3(\mathrm{RR}=1.944 ; \mathrm{P}=0.008)$ were independent prognostic factors for shorter DFS time. TNM stage $(\mathrm{HR}=9.75 ; \mathrm{P}<0.001)$ and histological grade $(\mathrm{HR}=2.592$; $\mathrm{P}=0.004)$ were independent prognostic factors for shorter OS in patients with breast cancer.

Association of PARP-3 expression with therapeutic responses in patients with breast cancer. The association between the level of PARP-3 expression and therapeutic responses in patients with breast cancer receiving chemotherapy was examined. PARP-3 overexpression was significantly associated with shorter DFS time in patients with breast cancer with $\mathrm{CAF} / \mathrm{CEF}$ treatment $(\mathrm{P}=0.006)$ (Fig. 5A), but not significantly associated with DFS $(\mathrm{P}=0.171)$ (Fig. $5 \mathrm{~B})$ in patients with $\mathrm{CAF} / \mathrm{CEF}+\mathrm{T}$ treatment. Although PARP-3 overexpression exhibited a tendency toward shorter OS in patients with breast cancer with $\mathrm{CAF} / \mathrm{CEF}$ treatment, no statistically significant difference was observed $(\mathrm{P}=0.088)$ (Fig. 5C). In addition, PARP-3 expression was not significantly associated with $\mathrm{OS}(\mathrm{P}=0.420)$ (Fig. 5D) in patients with $\mathrm{CAF} / \mathrm{CEF}+\mathrm{T}$ treatment.

\section{Discussion}

It is well known that DNA damage, if not repaired properly, can lead to genetic instability, which may increase the development of cancer (25). Previous studies have shown that PARP-3 serves an important role in DSB repair $(7,19)$. However, the role of PARP-3 in breast cancer tumorigenesis remains to be determined. In the present study, tissue-microarray-based immunohistochemistry was performed to examine PARP-3 expression in 493 patients with breast cancer and 54 tumor-adjacent control samples. It was revealed that PARP-3 immunoreactivity occurred more frequently in breast cancer samples compared with control samples, indicating that PARP-3 overexpression may contribute to the development of breast cancer malignancy. Similarly, several studies have shown that PARP-1, the most studied member of PARP superfamily that is also involved in DNA damage repair, is upregulated in numerous tumors, including breast cancer (8-13). These findings indicated that the DNA damage repair function of PARP-1 and PARP-3 may be important for cancer development. However, Bieche et al (20) reported that the mRNA expression of PARP-3 was under-expressed in $10.4 \%$ of patients with breast cancer. The difference between the study by Bieche et al and the present study may be due to different methods, since the 
A

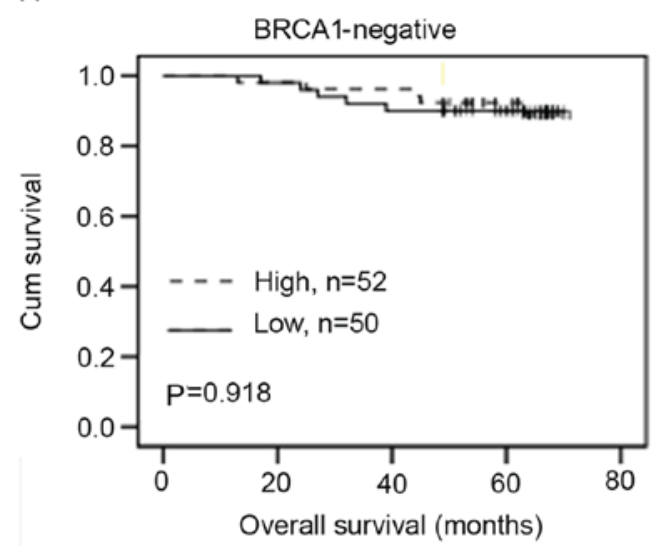

C

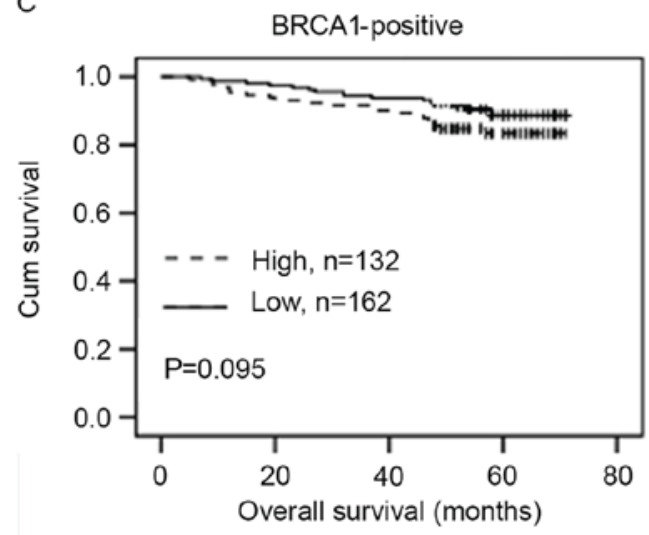

E

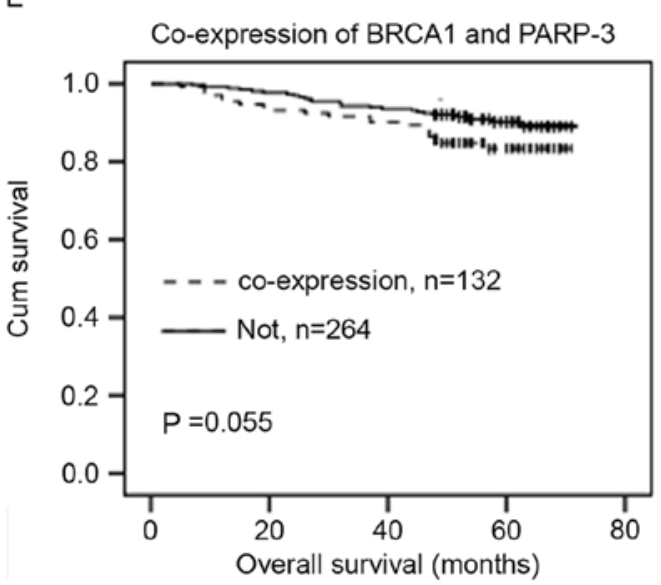

B

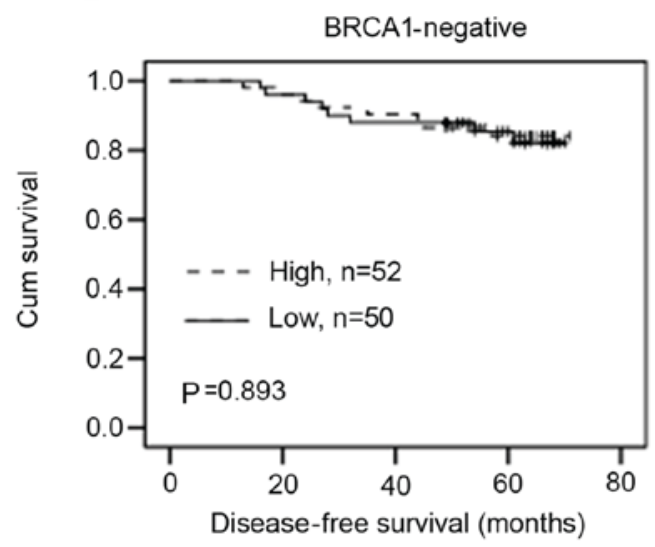

D

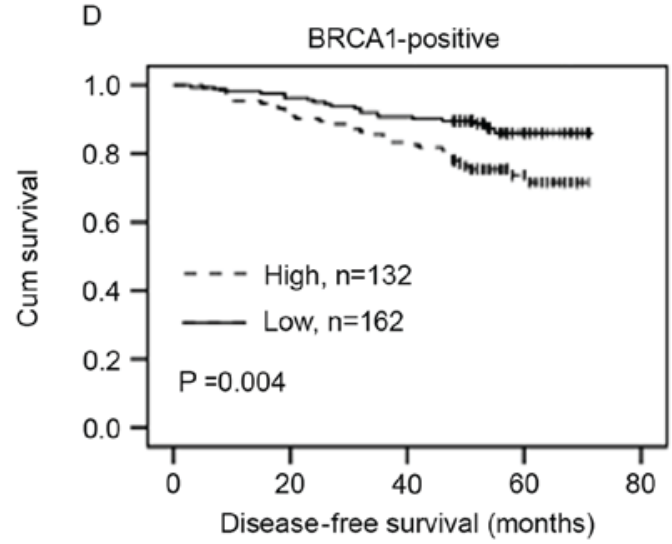

$\mathrm{F}$

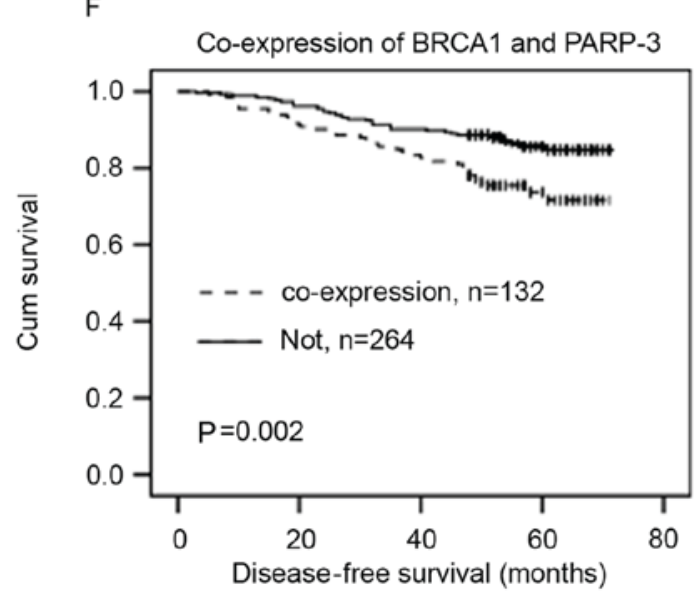

Figure 4. Kaplan-Meier survival analysis of PARP-3 expression in patients with breast cancer with different BRCA1 status. The log-rank test was performed to test statistical significance. (A-D) Survival curves show the association between PARP-3 expression and DFS or OS in patients with BRCA1-negative andBRCA1-positive breast cancer. (E and F) Survival curves show the association of the combined expression of PARP-3 and BRCA1 with OS or DFS in patients with breast cancer. PARP-3, poly(ADP-ribose) polymerase-3; DFS, disease-free survival; OS, overall-survival; BRCA1, breast cancer susceptibility gene 1; Cum, cumulative.

mRNA levels of PARP-3 detected by Bieche et al may not reflect the protein level of PARP-3 examined by the present study.

The association between PARP-3 expression and clinicopathological features in patients with breast cancer was analyzed in the present study. It was revealed that PARP-3 overexpression was associated with more differentiated (histological grade I-II) tumors. It is likely that PARP-3 is upregulated in response to an increase in DNA breaks during tumor cell differentiation, and PARP-3 upregulation may promote repair of DNA damage in tumors, thereby increasing cancer progression and development. Consistent with this hypothesis, PARP-3 overexpression was also found to be significantly associated with shorter DFS time and exhibited a tendency toward shorter OS in patients with breast cancer. Similarly, PARP-1 overexpression has been reported to be associated with poor prognosis in patients with breast cancer $(12,26)$. Furthermore, it was found that PARP-3 overexpression is an independent prognostic factor for shorter DFS and OS in patients with breast cancer. 
Table III. Univariate Cox regression analysis of overall survival and disease-free survival in patients with breast cancer.

\begin{tabular}{|c|c|c|c|c|}
\hline \multirow[b]{2}{*}{ Parameters } & \multicolumn{2}{|c|}{ DFS } & \multicolumn{2}{|c|}{ OS } \\
\hline & HR (95\% CI) & P-value ${ }^{a}$ & HR $(95 \%$ CI $)$ & P-value ${ }^{a}$ \\
\hline Age $(>51 / \leq 51$ years $)$ & $1.352(0.90-2.04)$ & 0.148 & $1.702(1.02-2.84)$ & 0.041 \\
\hline Menopausal status (post/pre) & $1.527(1.01-2.30)$ & 0.044 & $1.846(1.10-3.09)$ & 0.020 \\
\hline Tumor size $(>2.0 / \leq 2.0 \mathrm{~cm})$ & $1.272(0.83-1.95)$ & 0.271 & $1.714(1.02-2.88)$ & 0.042 \\
\hline Histological grade (III/II/I) & $2.674(1.24-5.78)$ & 0.012 & $6.350(1.55-25.99)$ & 0.010 \\
\hline Histological type (ductal/lobular) & $0.804(0.53-1.21)$ & 0.296 & $0.569(0.26-1.23)$ & 0.153 \\
\hline TNM stage (V/IV/III/II/I) & $4.200(2.78-6.34)$ & $<0.001$ & $9.084(5.12-16.11)$ & $<0.001$ \\
\hline Lymph node status (yes/no) & $2.513(1.63-3.87)$ & $<0.001$ & $4.572(2.47-8.45)$ & $<0.001$ \\
\hline BRCA1 status (positive/negative) & $1.365(0.79-2.38)$ & 0.271 & $1.431(0.71-2.87)$ & 0.312 \\
\hline p53 status (positive/negative) & $1.063(0.67-1.68)$ & 0.792 & $1.027(0.59-1.79)$ & 0.927 \\
\hline PARP-3 (positive/negative) & $1.619(1.05-2.49)$ & 0.029 & $1.434(0.84-2.44)$ & 0.186 \\
\hline
\end{tabular}

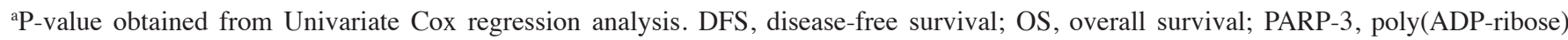
polymerase-3; TNM, tumor-node-metastasis; BRCA1, breast cancer susceptibility gene 1; HR, hazard ratio; 95\% CI, 95\% confidence interval.

Table IV. Multivariate Cox regression analysis of overall survival and disease-free survival in breast in patients with breast cancer.

\begin{tabular}{|c|c|c|c|c|}
\hline \multirow[b]{2}{*}{ Category } & \multicolumn{2}{|c|}{ Disease-free survival } & \multicolumn{2}{|c|}{ Overall survival } \\
\hline & $\mathrm{RR}(95 \% \mathrm{CI})$ & P-value ${ }^{a}$ & $\mathrm{RR}(95 \% \mathrm{CI})$ & P-value \\
\hline $\operatorname{Age}(>51 / \leq 51$ years $)$ & $0.740(0.30 \sim 1.82)$ & 0.513 & $0.77(0.215 \sim 2.76)$ & 0.689 \\
\hline Menopausal status (post/pre) & $2.535(1.02 \sim 6.30)$ & 0.045 & $3.156(0.85 \sim 11.70)$ & 0.086 \\
\hline Tumor size $(\geq 5 / 2-5 / \leq 2.0 \mathrm{~cm})$ & $1.163(0.77 \sim 1.77)$ & 0.481 & $1.543(0.95 \sim 2.51)$ & 0.081 \\
\hline Histological grade (III/II/I) & $1.681(0.98 \sim 2.90)$ & 0.062 & $2.592(1.35 \sim 4.97)$ & 0.004 \\
\hline Histological type (ductal/ lobular/mucinous/other) & $0.952(0.52-1.76)$ & 0.875 & $0.591(0.14-2.52)$ & 0.477 \\
\hline TNM stage (V/IV/III/II/I) & $5.665(2.56 \sim 12.5)$ & $<0.001$ & $9.75(3.12 \sim 30.51)$ & $<0.001$ \\
\hline Lymph node status $(\geq 10 / 4 \sim 9 / 1 \sim 3 / 0)$ & $0.720(0.31 \sim 1.65)$ & 0.439 & $0.934(0.26 \sim 3.35)$ & 0.917 \\
\hline PARP-3 (positive/negative) & $1.944(1.19 \sim 3.19)$ & 0.008 & $1.716(0.93 \sim 3.15)$ & 0.082 \\
\hline BRCA1 status (positive/negative) & $1.167(0.64 \sim 2.12)$ & 0.612 & $1.078(0.51 \sim 2.29)$ & 0.846 \\
\hline p53 status (positive/negative) & $1.130(0.69 \sim 1.87)$ & 0.632 & $1.116(0.60 \sim 2.08)$ & 0.730 \\
\hline
\end{tabular}

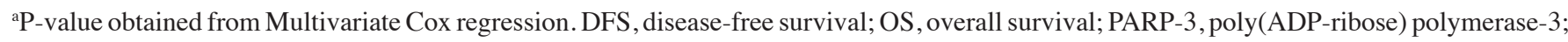
TNM, tumor-node-metastasis; RR, relative risk; $95 \% \mathrm{CI}, 95 \%$ confidence interval.

Therefore, PARP-3 may be used as a potential biomarker for clinical outcomes of patients with breast cancer.

Eukaryotic cells have two repair pathways to repair DSB: Homologous recombination and non-homologous end joining (NHEJ) (27). PARP-3 in combination with aprataxin and PNKP-like factor accelerates NHEJ $(28,29)$, and BRCA1 is known to be a central component in homologous recombination (30-32). Furthermore, Beck et al reported that PARP-3 performs a key role in determining the choice between homologous recombination and NHEJ pathways in the repair of DSB (33). In the present study, it was indicated that PARP-3 overexpression was significantly associated with shorter DFS time and exhibited a tendency toward shorter OS in BRCA1-positive patients with breast cancer. Additionally, the combined high expression of BRCA1 and PARP-3 was associated with shorter DFS and OS in patients with breast cancer compared with non-combined BRCA1 and PARP-3 expression. Therefore, for tumor cells with high expression of BRCA1, PARP-3-overexpressing cells may able to repair DNA damage more efficiently compared with PARP-3 deficient cells, thus leading to prolonged survival of the tumor and poor prognosis of the patients with cancer. However, it was found that PARP-3 expression was not significantly associated with the OS or DFS in BRCA1-negative patients with breast cancer. The findings that PARP-3 overexpression was significantly associated with the shorter survival time in BRCA1-positive, but not BRCA1-negative, breast cancer patients suggest that the role of PARP-3 in breast cancer may depend on BRCA1 status. Therefore, PARP-3 inhibitor may be a novel strategy for the treatment of BRAC1-positive breast cancer patients with PARP-3 overexpression. 
A

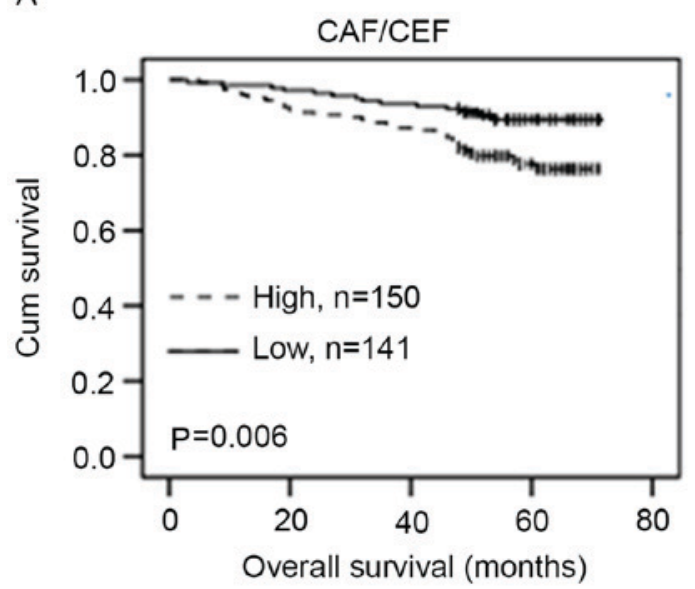

C

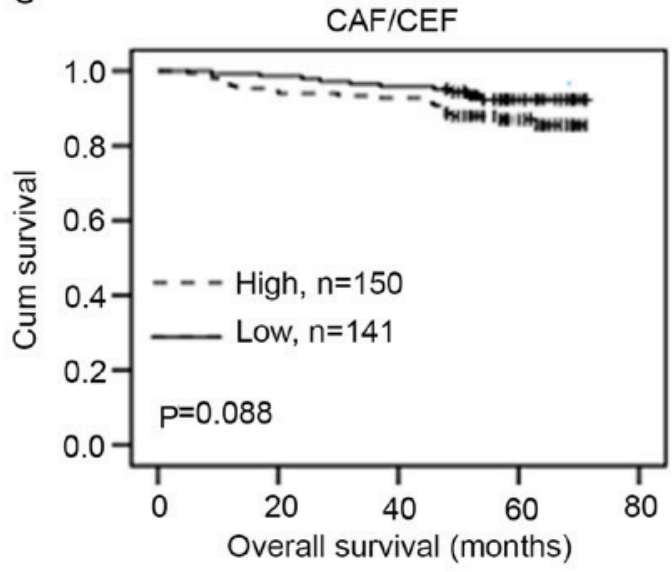

B
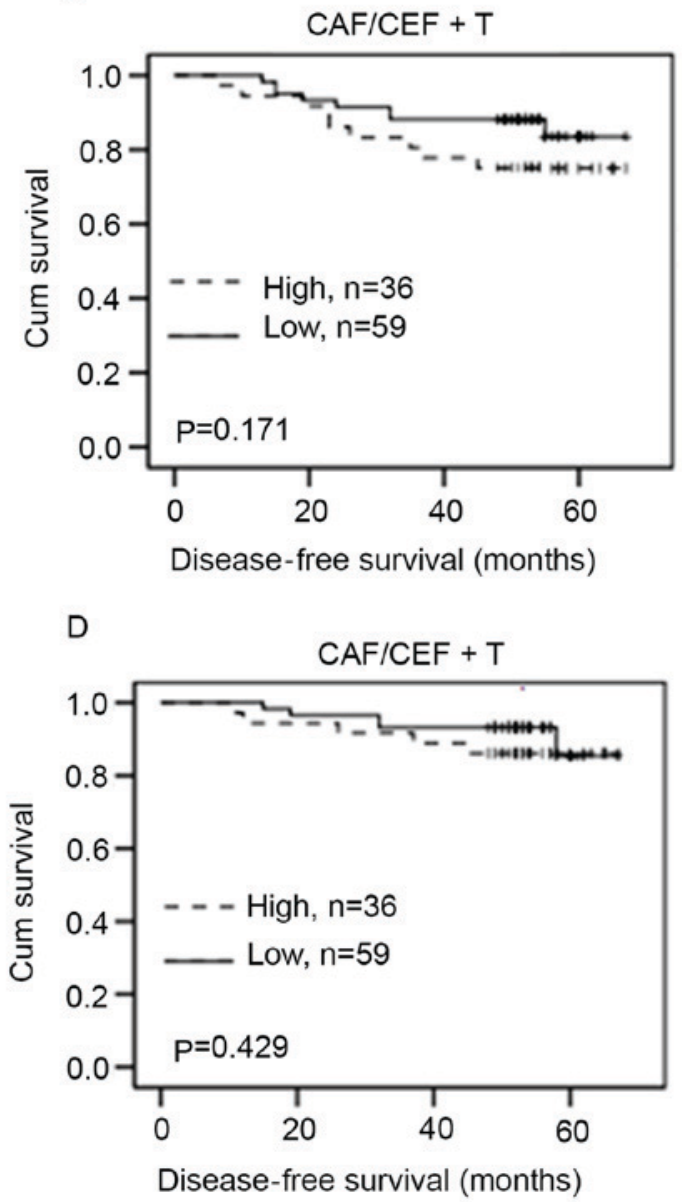

Figure 5. Kaplan-Meier survival analysis of PARP-3 expression in patients with breast cancer with chemotherapy. (A-D) Survival curves show the association between PARP-3 expression and disease-free survival oroverall survival in patients with breast cancer receiving $\mathrm{CAF} / \mathrm{CEF}$ treatment or $\mathrm{CAF} / \mathrm{CEF}+\mathrm{T}$ treatment. PARP-3, poly(ADP-ribose) polymerase-3; CAF/CEF, cyclophosphamide/doxorubicin or epirubicin/5-fluorouracil; $\mathrm{CAF} / \mathrm{CEF}+\mathrm{T}, \mathrm{CAF} / \mathrm{CEF}+\mathrm{docetaxel}$.

It is known that chemotherapeutic drugs can induce DNA damage, and DNA damage repair may affect the outcome of therapy (34). In the present study, the association of PARP-3 expression with therapeutic responses in patients with breast cancer receiving $\mathrm{CAF} / \mathrm{CEF}$ chemotherapy was examined. It was revealed that PARP-3 overexpression was significantly associated with shorter DFS time, and exhibited a tendency toward shorter OS in patients with breast cancer who received $\mathrm{CAF} / \mathrm{CEF}$ treatment. The present findings suggested that tumors with PARP-3 overexpression exhibited resistance to chemotherapy, possibly by an increased ability of DNA repair. Furthermore, PARP-3 overexpression was not significantly associated with DFS and OS in patients with CAF/CEF+T treatment. It appears that addition of docetaxel inhibited PARP-3-induced drug resistance in patients with breast cancer. It has been reported that docetaxel can cause cleavage of PARP in breast cancer cells, melanoma cells and ovarian cancer cells (35-37). Taken together, the present results suggested that docetaxel may induce cleavage of PARP-3, thereby reducing PARP-3-induced drug resistance and improving the survival of patients with breast cancer with PARP-3 overexpression.

In summary, PARP-3 expression was investigated in 493 patients with breast cancer, and the association of PARP-3 expression with the clinicopathological feature, therapeutic responses and prognosis of patients with breast cancer was analyzed. It was found that PARP-3 expression was significantly increased in breast cancer tissues compared with tumor-adjacent tissues. PARP-3 overexpression was associated with poor outcome of patients with breast cancer, particularly in BRCA1-positive patients. Furthermore, it was found that PARP-3 overexpression was associated with shorter survival time in patients with $\mathrm{CAF} / \mathrm{CEF}$ chemotherapy, but not in patients with $\mathrm{CAF} / \mathrm{CEF}+\mathrm{T}$ chemotherapy, indicating that inhibition of PARP-3 by docetaxel may increase the survival of patients with breast cancer. PARP-3 may be used as a biomarker for predicting the clinical outcome of patients receiving chemotherapy, and targeting PARP-3 may be a potential therapeutic strategy for the treatment of breast cancer with PARP-3 overexpression.

\section{Acknowledgements}

The authors would like to acknowledge Xuefeng Bai for providing technical help and Xiaosong Yu for assisting in writing the manuscript.

\section{Funding}

The present study was supported by grants from the National Natural Science Foundation of the People's Republic of China (grant no. 71273279; Beijing China), Program for Liaoning 
Innovative Research Team in University, (grant no. LT2014016; Shenyang, China) and Shenyang Science and Technology Projects (grant no. F14-232-6-05; Shenyang, China).

\section{Availability of data and materials}

The datasets used and/or analyzed during the current study are available from the corresponding author on reasonable request.

\section{Authors' contribution}

MW, ZS, LZ and HW assisted in the design and conception of the present study. ZS, YW, QX, MS and ZY analyzed and interpreted the data of patients with breast cancer. ZS, WL and $\mathrm{XG}$ performed the histological examination of the breast cancer tissues. LZ, HW, MS contributed in drafting the manuscript. QX and MW were major contributor in revising the manuscript. ZC, PH collected the patients' clinical data and performed the follow-up study. All authors read and approved the final manuscript.

\section{Ethics approval and consent to participate}

The Institute Research Medical Ethics Committee of China Medical University approved the consent procedure.

\section{Patient consent for publication}

The patient, or parent, guardian or next of kin (if patient is deceased) provided verbal informed consent for the publication of any associated data and accompanying images.

\section{Competing interests}

The authors declare that they have no competing interests.

\section{References}

1. Bertucci F and Birnbaum D: Reasons for breast cancer heterogeneity. J Biol 7: 6, 2008.

2. Patani N, Martin LA and Dowsett M: Biomarkers for the clinical management of breast cancer: International perspective. Int J Cancer 133: 1-13, 2013.

3. Hakmé A, Wong HK, Dantzer F and Schreiber V: The expanding field of poly(ADP-ribosyl)ation reactions. 'Protein modifications: Beyond the usual suspects' review series. EMBO Rep 9: 1094-1100, 2008.

4. Hottiger MO, Hassa PO, Lüscher B, Schüler H and Koch-Nolte F: Toward a unified nomenclature for mammalian ADP-ribosyltransferases. Trends Biochem Sci 35: 208-219, 2010.

5. Megnin-Chanet F, Bollet MA and Hall J: Targeting poly(ADP-ribose) polymerase activity for cancer therapy. Cell Mol Life Sci 67: 3649-3662, 2010.

6. Cepeda V, Fuertes MA, Castilla J, Alonso C, Quevedo C, Soto M and Pérez JM: Poly(ADP-ribose) polymerase-1 (PARP-1) inhibitors in cancer chemotherapy. Recent Pat Anticancer Drug Discov 1: 39-53, 2006.

7. Boehler C and Dantzer F: PARP-3, a DNA-dependent PARP with emerging roles in double-strand break repair and mitotic progression. Cell Cycle 10: 1023-1024, 2011.

8. Miwa M and Masutani M: PolyADP-ribosylation and cancer. Cancer Sci 98: 1528-1535, 2007.

9. Shimizu S, Nomura F, Tomonaga T, Sunaga M, Noda M, Ebara M and Saisho H: Expression of poly(ADP-ribose) polymerase in human hepatocellular carcinoma and analysis of biopsy specimens obtained under sonographic guidance. Oncol Rep 12: 821-825, 2004.
10. Staibano S, Pepe S, Lo Muzio L, Somma P, Mascolo M, Argenziano G, Scalvenzi M, Salvatore G, Fabbrocini G, Molea G, et al: Poly(adenosine diphosphate-ribose) polymerase 1 expression in malignant melanomas from photoexposed areas of the head and neck region. Hum Pathol 36: 724-731, 2005.

11. Brustmann H: Poly(adenosine diphosphate-ribose) polymerase expression in serous ovarian carcinoma: Correlation with $\mathrm{p} 53$, MIB-1, and outcome. Int J Gynecol Pathol 26: 147-153, 2007.

12. Rojo F, García-Parra J, Zazo S, Tusquets I, Ferrer-Lozano J, Menendez S, Eroles P, Chamizo C, Servitja S, Ramírez-Merino N, et al: Nuclear PARP-1 protein overexpression is associated with poor overall survival in early breast cancer. Ann Oncol 23: 1156-1164, 2012.

13. Ossovskaya V, Koo IC, Kaldjian EP, Alvares C and Sherman BM: Upregulation of Poly(ADP-Ribose) Polymerase-1 (PARP1) in triple-negative breast cancer and other primary human tumor types. Genes Cancer 1: 812-821, 2010.

14. O'Shaughnessy J, Osborne C, Pippen JE, Yoffe M, Patt D, Rocha C, Koo IC, Sherman BM and Bradley C: Iniparib plus chemotherapy in metastatic triple-negative breast cancer. N Engl J Med 364: 205-214, 2011.

15. Fong PC, Boss DS, Yap TA, Tutt A, Wu P, Mergui-Roelvink M, Mortimer P, Swaisland H, Lau A, O'Connor MJ, et al: Inhibition of poly(ADP-ribose) polymerase in tumors from BRCA mutation carriers. N Engl J Med 361: 123-134, 2009.

16. Tutt A, Robson M, Garber JE, Domchek SM, Audeh MW, Weitzel JN, Friedlander M, Arun B, Loman N, Schmutzler RK, et al: Oral poly(ADP-ribose) polymerase inhibitor olaparib in patients with BRCA1 or BRCA 2 mutations and advanced breast cancer: A proof-of-concept trial. Lancet 376: 235-244, 2010.

17. De Vos M, Schreiber V and Dantzer F: The diverse roles and clinical relevance of PARPs in DNA damage repair: Current state of the art. Biochem Pharmacol 84: 137-146, 2012.

18. Langelier MF, Riccio AA and Pascal JM: PARP-2 and PARP-3 are selectively activated by 5 ' phosphorylated DNA breaks through an allosteric regulatory mechanism shared with PARP-1. Nucleic Acids Res 42: 7762-7775, 2014.

19. Boehler C, Gauthier LR, Mortusewicz O, Biard DS, Saliou JM, Bresson A, Sanglier-Cianferani S, Smith S, Schreiber V, Boussin F and Dantzer F: Poly(ADP-ribose) polymerase 3 (PARP3), a newcomer in cellular response to DNA damage and mitotic progression. Proc Natl Acad Sci USA 108: 2783-2788, 2011.

20. Bieche I, Pennaneach V, Driouch K, Vacher S, Zaremba T, Susini A, Lidereau R and Hall J: Variations in the mRNA expression of poly(ADP-ribose) polymerases, poly(ADP-ribose) glycohydrolase and ADP-ribosylhydrolase 3 in breast tumors and impact on clinical outcome. Int J Cancer 133: 2791-2800, 2013.

21. Bansal C, Pujani M, Sharma KL, Srivastava AN and Singh US: Grading systems in the cytological diagnosis of breast cancer: A review. J Cancer Res Ther 10: 839-845, 2014.

22. Cowherd SM: Tumor staging and grading: A primer. Methods Mol Biol 823: 1-18, 2012.

23. Fang Y, Wei J, Cao J, Zhao H, Liao B, Qiu S, Wang D, Luo J and Chen W: Protein expression of ZEB2 in renal cell carcinoma and its prognostic significance in patient survival. PLoS One 8: e62558, 2013.

24. Zhu W, Cai MY, Tong ZT, Dong SS, Mai SJ, Liao YJ, Bian XW, Lin MC, Kung HF, Zeng YX, et al: Overexpression of EIF5A2 promotes colorectal carcinoma cell aggressiveness by upregulating MTA1 through C-myc to induce epithelial-mesenchymaltransition. Gut 61: 562-575, 2012.

25. Khanna KK and Jackson SP: DNA double-strand breaks: Signaling, repair and the cancer connection. Nat Genet 27: 247-254, 2001.

26. Goncalves A, Finetti P, Sabatier R, Gilabert M, Adelaide J, Borg JP, Chaffanet M, Viens P, Birnbaum D and Bertucci F: Poly(ADP-ribose) polymerase-1 mRNA expression in human breast cancer: A meta-analysis. Breast Cancer Res Treat 127: 273-281, 2011.

27. Chapman JR, Taylor MR and Boulton SJ: Playing the end game: DNA double-strand break repair pathway choice. Mol Cell 47: 497-510, 2012.

28. Rulten SL, Fisher AE, Robert I, Zuma MC, Rouleau M, Ju L, Poirier G, Reina-San-Martin B and Caldecott KW: PARP-3 and APLF function together to accelerate nonhomologous end-joining. Mol Cell 41: 33-45, 2011.

29. Fenton AL, Shirodkar P, Macrae CJ, Meng L and Koch CA: The PARP3- and ATM-dependent phosphorylation of APLF facilitates DNA double-strand break repair. Nucleic Acids Res 41: 4080-4092, 2013. 
30. Zhang J: The role of BRCA1 in homologous recombination repair in response to replication stress: Significance in tumorigenesis and cancer therapy. Cell Biosci 3: 11, 2013.

31. Prakash R, Zhang Y, Feng W and Jasin M: Homologous recombination and human health: The roles of BRCA1, BRCA2, and associated proteins. Cold Spring Harb Perspect Biol 7: a016600, 2015.

32. Willis NA, Chandramouly G, Huang B, Kwok A, Follonier C, Deng $\mathrm{C}$ and Scully R: BRCA1 controls homologous recombination at Tus/Ter-stalled mammalian replication forks. Nature 510 556-559, 2014.

33. Beck C, Boehler C, Guirouilh Barbat J, Bonnet ME, Illuzzi G, Ronde P, Gauthier LR, Magroun N, Rajendran A, Lopez BS, et al: PARP3 affects the relative contribution of homologous recombination and nonhomologous end-joining pathways. Nucleic Acids Res 42: 5616-5632, 2014.

34. Casorelli I, Bossa C and Bignami M: DNA damage and repair in human cancer: Molecular mechanisms and contribution to therapy-related leukemias. Int J Environ Res Public Health 9: 2636-2657, 2012.

35. Wang Q and Wieder R: All-trans retinoic acid potentiates Taxotere-induced cell death mediated by Jun $\mathrm{N}$-terminal kinase in breast cancer cells. Oncogene 23: 426-433, 2004.
36. Mhaidat NM, Wang Y, Kiejda KA, Zhang XD and Hersey P: Docetaxel-induced apoptosis in melanoma cells is dependent on activation of caspase-2. Mol Cancer Ther 6: 752-761, 2007.

37. Kolfschoten GM, Hulscher TM, Duyndam MC, Pinedo HM and Boven E: Variation in the kinetics of caspase-3 activation, Bcl-2 phosphorylation and apoptotic morphology in unselected human ovarian cancer cell lines as a response to docetaxel. Biochem Pharmacol 63: 733-743, 2002.

(i) (9) This work is licensed under a Creative Commons Attribution-NonCommercial-NoDerivatives 4.0 International (CC BY-NC-ND 4.0) License. 